\title{
Subcorneal Pustular Dermatosis Exhibiting a High Serum TARC/CCL17 Level
}

\author{
Sachiko Ono Atsushi Otsuka Yoshiki Miyachi Kenji Kabashima \\ Department of Dermatology, Kyoto University Graduate School of Medicine, Kyoto, Japan
}

\section{Key Words}

Subcorneal pustular dermatosis $\cdot$ Neutrophilic dermatosis $\cdot$ TARC/CCL17 $\cdot$ T helper 2

\begin{abstract}
Subcorneal pustular dermatosis (SPD) is a rare, relapsing, symmetric sterile pustular eruption that dominantly involves flexural areas. It is considered one form of neutrophilic dermatoses, which are associated with interleukin (IL)-17-producing Thelper (Th) 17 cells that induce IL-8 production. We have previously reported that Th17 might be involved in the pathomechanism of Th2-dominant atopic dermatitis [Koga et al.: J Invest Dermatol 2008;128:2625-2630]. On the other hand, it has been a debate whether Th2 is involved in the etiology of neutrophilic dermatoses. Herein, we report a case of SPD that exhibited a high serum thymus and activation-regulated chemokine/chemokine (C-C motif) ligand 17 level, thereby raising the possibility of a Th2 association in its pathogenesis. Although it was limited to a single observation, our case raised the possibility that SPD may possess Th2 properties.
\end{abstract}

\section{Introduction}

Subcorneal pustular dermatosis (SPD) is a rare, relapsing, symmetric sterile pustular eruption that dominantly involves flexural areas. It was first described by Sneddon and Wilkinson in 1956 [1], as mentioned in the study by Cheng et al. [2]. It is considered to be one form of neutrophilic dermatoses belonging to a similar category as Sweet's syndrome, generalized pustular psoriasis (GPP), and acute generalized exanthematous pustulosis (AGEP). These neutrophilic dermatoses are considered to be induced by interleukin (IL)-8, which attracts neutrophils [3]. The production of IL-8 is stimulated by IL-17-producing cells, such as Thelper (Th) 17 cells [4]. 
Thymus and activation-regulated chemokine/chemokine (C-C motif) ligand 17 (TARC/CCL17) is designated as a Th2 chemokine, the serum level of which reflects the disease activity of Th2-mediated diseases, such as atopic dermatitis, mycosis fungoides, and bullous pemphigoid [5]. It remains unclear whether Th2 conditions are related to the pathogenesis of neutrophilic dermatoses. Herein, we report a case of SPD that exhibited a high serum TARC/CCL17 level, thereby raising the possibility of both Th17 and Th2 association in its pathogenesis.

\section{Case Presentation}

A 65-year-old man presented with pruritic generalized erythematous plaques with scales and small pustules that had been present for 6 years. The eruptions covered $30-40 \%$ of his body surface (fig. 1). The eruptions occasionally fluctuated, and the general condition of the patient was quite good throughout this time period. Laboratory examinations revealed no abnormal findings except for an elevated serum TARC/CCL17 level of $6,375 \mathrm{pg} / \mathrm{ml}$ (control, <450). White blood cell counts $\left(6.5 \times 10^{9} / \mathrm{l}\right)$, neutrophil counts $\left(3.5 \times 10^{9} / \mathrm{l}\right)$, eosinophil counts $\left(0.39 \times 10^{9} / \mathrm{l}\right)$, and C-reactive protein $(<0.3 \mathrm{mg} / \mathrm{dl})$ were within normal range. The patient was otherwise healthy and did not have a past history of psoriasis vulgaris or atopic dermatitis. He had no medication intake or family history. A computed tomography examination revealed no malignancy. A histological examination revealed a subcorneal neutrophilic pustule, epidermal acanthosis, lymphocytic infiltrations into the epidermis, and mild lymphocytic infiltrations into the upper dermis (fig. 2).

Differential diagnoses of GPP, SPD, and AGEP were considered clinically and histologically from the subcorneal pustules. However, there were no apparent Kogoj's spongiform pustules, systemic symptoms, or laboratory abnormalities to fulfill the diagnostic criteria for GPP. AGEP was unlikely since the patient was medication free. Therefore, the patient was diagnosed as SPD without underlying disorders.

What is intriguing in our case is the high serum TARC/CCL17 level observed under the neutrophilic inflammation status of SPD. As mentioned above, neutrophilic dermatosis is associated with IL-17-producing Th17 that induces IL-8 production [4]. Although the elevation of IL-17 in the skin lesion of atopic dermatitis that is considered as a typical Th2mediated skin disease [6], the association between Th17 and Th2 environments in the development of neutrophilic dermatoses has not yet been well documented. Thus far, only a single case of an elevated serum TARC/CCL17 level in AGEP has been reported [7]. It is of note that Th17 cells enhance not only neutrophilic inflammation but also Th2 cell-mediated inflammation in a mouse model of asthma [8]. In addition, the barrier dysfunction in SPD may induce the expression of thymic stromal lymphopoietin on keratinocytes, which may in turn induce the TARC/CCL17 expression and Th2 conditions [9]. Although it was limited to a single observation, our case also raised the possibility that SPD may possess Th2 properties. Moreover, an elevated tumor necrosis factor- $\alpha$ condition in SPD may enhance the synthesis of TARC/CCL17 from keratinocytes [10]. Further investigation and accumulation of serum TARC/CCL17 levels in cases of neutrophilic dermatosis is warranted.

\section{Disclosure Statement}

The authors declare that there are no conflicts of interest regarding this paper. 


\section{References}

1 Sneddon IB, Wilkinson DS: Subcorneal pustular dermatosis. Br J Dermatol 1956;68:385-394.

-2 Cheng S, Edmonds E, Ben-Gashir M, Yu RC: Subcorneal pustular dermatosis: 50 years on. Clin Exp Dermatol 2008;33:229-233.

-3 Keller M, Spanou Z, Schaerli P, Britschgi M, Yawalkar N, Seitz M, Villiger PM, Pichler WJ: T cell-regulated neutrophilic inflammation in autoinflammatory diseases. J Immunol 2005;175:7678-7686.

-4 Nakamizo S, Kobayashi S, Usui T, Miyachi Y, Kabashima K: Clopidogrel-induced acute generalized exanthematous pustulosis with elevated Th17 cytokine levels as determined by a drug lymphocyte stimulation test. Br J Dermatol 2010;162:1402-1403.

-5 Kagami S, Sugaya M, Minatani Y, Ohmatsu H, Kakinuma T, Fujita H, Tamaki K: Elevated serum CTACK/CCL27 levels in CTCL. J Invest Dermatol 2006;126:1189-1191.

6 Koga C, Kabashima K, Shiraishi N, Kobayashi M, Tokura Y: Possible pathogenic role of Th17 cells for atopic dermatitis. J Invest Dermatol 2008;128:2625-2630.

-7 Cançado GG, Fujiwara RT, Freitas PA, Correa-Oliveira R, Bethony JM: Acute generalized exanthematous pustulosis induced by itraconazole: an immunological approach. Clin Exp Dermatol 2009;34:e709-e711.

-8 Wakashin H, Hirose K, Maezawa Y, Kagami S, Suto A, Watanabe N, et al: IL-23 and Th17 cells enhance Th2cell-mediated eosinophilic airway inflammation in mice. Am J Respir Crit Care Med 2008;178:1023-1032.

-9 Nakajima S, Igyártó BZ, Honda T, Egawa G, Otsuka A, Hara-Chikuma M, et al: Langerhans cells are critical in epicutaneous sensitization with protein antigen via thymic stromal lymphopoietin receptor signaling. J Allergy Clin Immunol 2012;129:1048-1055.e1046.

10 Yu B, Koga T, Urabe K, Moroi Y, Maeda S, Yanagihara Y, Furue M: Differential regulation of thymus- and activation-regulated chemokine induced by IL-4, IL-13, TNF-alpha and IFN-gamma in human keratinocyte and fibroblast. J Dermatol Sci 2002;30:29-36. 

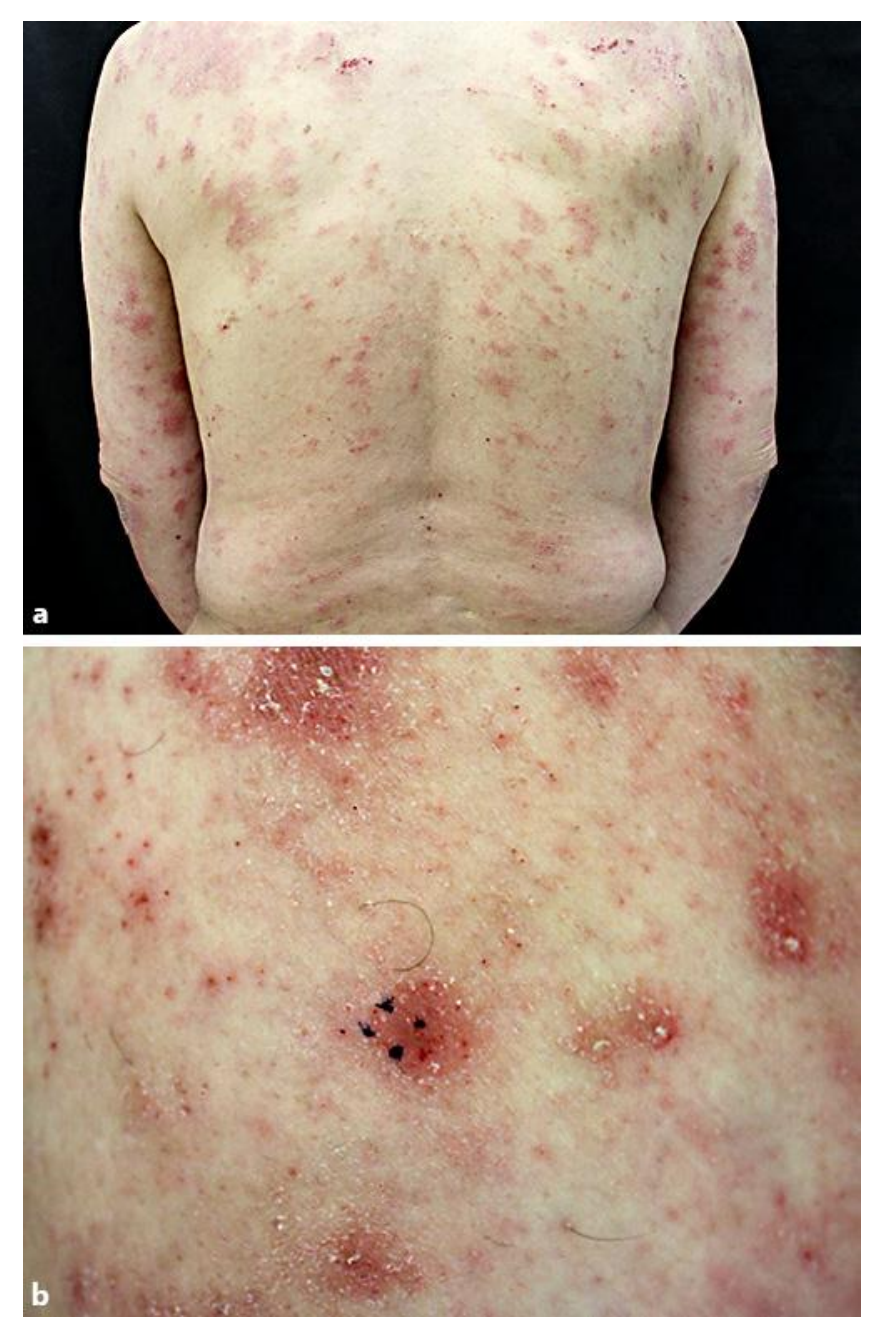

Fig. 1. Clinical images. a Generalized erythematous plaques with scales and small pustules were observed. b A biopsy specimen was taken from the right thigh. 


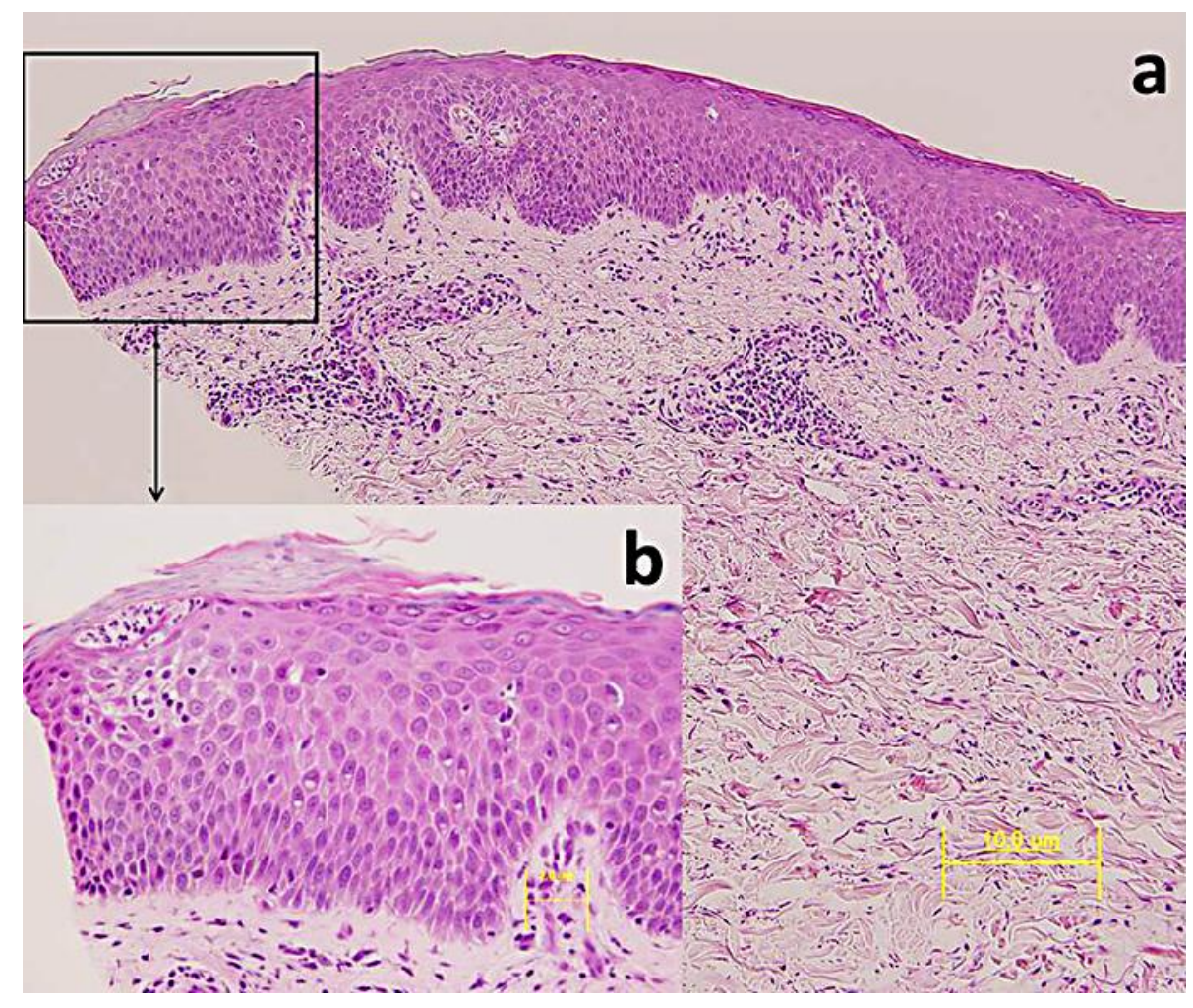

Fig. 2. Histological findings. a A neutrophilic subcorneal pustule, epidermal acanthosis, lymphocytic infiltrations into the epidermis, and mild lymphocytic infiltrations in the upper dermis were observed. Bar: $10 \mu \mathrm{m}$. b Magnified view of a subcorneal pustule. Bar: $2 \mu \mathrm{m}$. 